\title{
Counting Moving Bodies Using Sparse Sensor Beams
}

Lawrence H. Erickson, Jingjin Yu, Yaonan Huang, and Steven M. LaValle

\begin{abstract}
This paper examines the problem of determining the distribution of a number of indistinguishable moving bodies located in regions separated by sensor beams that can detect whether a body moves across them. We characterize the conditions under which an exact distribution of bodies can be determined, and compute bounds on the expected number of sensor observations required to determine this exact distribution for a certain movement model of the bodies.
\end{abstract}

\section{Introduction}

Consider determining, in a large office building with many rooms, how its anonymous occupants are scattered in the rooms. Such information can be of vital importance in scenarios such as coordinated building evacuation in an emergency or characterizing building usage for energy optimization. Let the distribution of the occupants be the precise number of occupants per room. The task is relatively easy if an initial distribution of the occupants is known: We may simply place sensor beams at doorways of rooms to figure out the change in population for each room as the occupants move around. Adding or subtracting the change from the initial count then yields the answer. But what if the a priori distribution of the occupants is unavailable? Could the task still be solved without additional sensors?

In this paper, we show that, somewhat surprisingly, the aforementioned simplistic sensor setup is still powerful enough for determining the occupant distribution as required, given only an initial total population and enough time. More precisely, we

Lawrence H. Erickson, Yaonan Huang, Steven M. LaValle

Department of Computer Science, University of Illinois at Urbana-Champaign,

e-mail: lericks4@uiuc.edu, huang134@illinois.edu, lavalle@uiuc.edu

Jingjin Yu

Department of Electrical and Computer Engineering, University of Illinois at Urbana-Champaign, e-mail: jyu18@uiuc.edu 


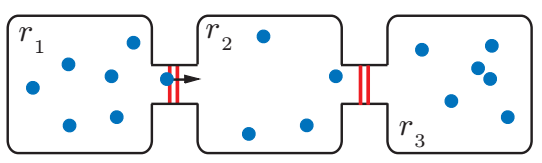

(a)

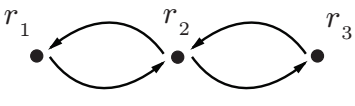

(b)

Fig. 1 a) A simple environment with three regions and two sensing units separating them, in which a blue body is moving from region $r_{1}$ to region $r_{2}$. b) The corresponding graph representation of the environment, induced by the sensor placement.

characterize the capabilities and limitations of using beams to keep track of bodies moving around in a closed and bounded environment partitioned into regions by these beams. A beam detector is perhaps one of the simplest sensors: As a fixed sensor, it detects that a moving body passes through it but it cannot determine any other property of the body. With two of these, which we call a sensing unit, it is also possible to tell the movement direction of the passing body. Fixing such sensing units between regions of interest, we have at any moment the net number of bodies that have moved in/out of any region. Figure 1(a) shows one of the simplest environments under this model. Note that the regions and sensing units can be effectively represented as vertices and edges of a (directed) graph (Figure 1(b)). The bodies occupy the vertices of the graph; each sensor observation corresponds to a crossing of a body over an (directed) edge of the graph.

The contributions of this paper are twofold. First, we determine a necessary and sufficient condition on the initial distribution of bodies and the sensor history that allows the determination of an exact count of the bodies in each region. Second, we determine bounds on the expected number of sensor observations required to acquire a count of the bodies in each region for a specific movement model of the bodies. We show that for some starting distributions, the expected number of sensor observations required to determine the distribution is exponential in the number of bodies, while for other starting distributions (even in the same graph), the expected number of observations required to determine a distribution is polynomial in the number of bodies.

Various simple sensor models have been investigated in the task of target tracking and counting. Binary proximity sensors have been employed to estimate positions and velocities of a moving body using particle filters [1] and moving averages [11]. The performance limit of a binary proximity sensor network in tracking a single target was discussed and approached in [13], followed by an extension to the tracking of multiple targets [14]. The task of counting multiple targets using simple sensors was also studied in [2], in which the problem of accurately counting the number of targets with overlapping footprints using pressure sensor arrays was solved with a novel approach of integrating over Euler characteristics. In the works mentioned so far, the sensor network's aggregate sensing range must cover the targets of interest at all times. When only a subset of an environment is guarded, word problems in groups $[4,5]$ naturally arise. For the setup in which targets moving inside a $2 \mathrm{D}$ region are monitored with a set of sensor beams, [15] studied the issues of possible target locations, target path reconstruction up to homotopy, and path winding 
numbers. Here, the surfacing of more interesting behaviors also induces an increase in computational complexity as well as convergence time, which is similar to what we observe in this paper. Controlling of sensorless wild bodies using various forms of gates was explored in [3], and some of the physically implemented gates used laser sensor beams to detect crossings between regions. Related to the control of sensorless wild bodies is the sensorless manipulation of polygonal parts studied in $[6,10]$.

If the limitation on using simple sensor models is lifted, research literature on target counting and tracking expands. Given the amount of papers and their diversified nature on this topic, we only mention a few of them. In [9] a simple virtual sensor, capable only of reporting visible features of the polygonal environment and the presence of indistinguishable targets, is able to count static targets using a minimal amount of data storage, though it is unclear how the simple virtual sensor could be implemented by simple physical sensors. In the domain of wireless sensor networks, the study of target counting and tracking is frequently coupled with communication efficiency and other concerns [7]. In [16], Simultaneous Localization and Mapping (SLAM) and Detection and Tracking of Moving Objects (DTMO) are combined to attack both problems at the same time. Real-time people counting with a network of image sensors is studied in [17].

The rest of the paper is organized as follows. Section 2 contains definitions that will be used throughout the paper. Section 3 describes the conditions under which the distribution of bodies can be determined. Section 4 describes a movement model for the bodies called the exponential random movement model. This section also describes a physical system that produces behavior closely approximated by the exponential random movement model. Section 5 contains an upper bound on the expected number of sensor observations required to count the bodies in each region when the behavior of the bodies matches the exponential random movement model. Section 6 contains a method of using the upper bound to estimate the number of bodies in the environment if that number is not known in advance. Section 7 contains a tight lower bound on the expected number of sensor observations required to count the number of bodies in each region when the behavior of the bodies matches the exponential random movement model. Section 8 contains results for a very simple 2-cycle environment. Section 9 discusses directions of future research.

\section{Definitions}

The vertices of an $r$-vertex directed graph $G$ containing no sinks ${ }^{1}$ are populated by a set $M$ of moving bodies, in which $|M|=m$. This graph represents the regions and sensor beams of a physical environment (see Figure 1). The bodies are capable of travelling through the edges into other vertices. When a body moves, a sensor observation $y$ is generated that identifies the edge that the body traversed. The system is in the $k$ th stage after the $k$ th sensor observation has been generated. Let $V(G)$ be 
the vertex set of graph $G$. A distribution is an assignment of the moving bodies to vertices of $G$.

We assume that no information about the initial distribution of moving bodies is known. The history information state $\mathcal{I}_{\text {hist }}(k)=\left[y_{1}, \ldots, y_{k}\right]$ is the list containing the first $k$ sensor observations. The bounds information state $\mathcal{I}_{\text {bounds }}(k)$ consists of two $r$-length vectors $\left[u_{1, k}, \ldots, u_{r, k}\right]$ (the upper bounds) and $\left[\ell_{1, k}, \ldots, \ell_{r, k}\right]$ (the lower bounds), where $u_{i, k}$ is the most bodies that could be in vertex $v_{i}$ in stage $k$ while remaining consistent with the previous sensor observations, and $\ell_{i, k}$ is the fewest bodies that could be in vertex $v_{i}$ at stage $k$ while remaining consistent with the previous sensor observations. The interval length length $\left(k, v_{i}\right)$ is the value of $u_{i, k}-\ell_{i, k}$ at stage $k$. A bounds information state with an interval length of 0 is called a counting information state. Once a counting information state has been reached, it is trivially easy to keep track of the number of bodies in each region. Since the bodies are indistinguishable to the sensors, a counting information state is an exact description of the system state. For some information state $I$, let $H(I)$ be the hypothesis set of $I$, defined as the set of distributions of bodies over the vertices that are consistent with the information state $I$.

When a distribution $d$ and lower bound set $L$ are presented in the form $(d, L)$, then the lower bound set $L$ is implied to be consistent with the distribution $d$ (in other words, there is no vertex $v_{i}$ in which $\ell_{i}$ is greater than the actual number of bodies in $\left.v_{i}\right)$. A distribution and lower bound set $(d, L)$ is near-complete for vertex $v_{i}$ if the lower bound $\ell_{j}$ is equal to the the number of bodies in $v_{j}$ for all $j \neq i$, and the number of bodies in $v_{i}$ is equal to $\ell_{i}+1$. Note that $(d, L)$ is near-complete if and only if $\sum \ell_{i}=m-1$.

\section{Counting moving bodies}

In this section, the goal is to characterize what types of initial distribution and information history combinations allow the determination of a counting information state. As a first step, we show that, as long as a count of the total number of bodies in the graph is known in advance, the lower bounds of the bounds information state are sufficient to represent all that is known about the distribution of the bodies.

Theorem 1. For all $k \in \mathbb{N}$ and all $v \in V(G)$, the interval length length $(k, v)=m-$ $\sum_{i=1}^{r} \ell_{i, k}$.

Proof. This will be shown inductively. As a base case, note that when $k=0$, all interval lengths are $m$ and $\ell_{i, 0}=0$ for all $1 \leq i \leq r$.

Assume that in stage $k-1$, all intervals had length $m-\sum_{i=1}^{r} \ell_{i, k-1}$. Let the $k$ th sensor observation be the transition of a body from $v_{s}$ to $v_{t}$. If $\ell_{s, k-1} \geq 1$ and $u_{t, k-1} \leq m-1$, then each hypothesis $h \in H\left(\mathcal{I}_{\text {bounds }}(k-1)\right)$ can be transformed into a distribution $h^{\prime}$ consistent with $\mathcal{I}_{\text {bounds }}(k-1)+y_{k}$ by removing one body from

\footnotetext{
${ }^{1}$ Graphs with sinks are excluded because bodies located in sinks are unable to move to different vertices, which renders them undetectable.
} 
$v_{s}$ (there must be at least one as $\ell_{s, k-1} \geq 1$ ) and adding a body to $v_{t}$ (there must be room for at least one more as $\left.u_{t, k-1} \leq m-1\right)$. Therefore, $\mathcal{I}_{\text {bounds }}(k)$ is constructed by setting $\ell_{i, k}=\ell_{i, k-1}$ and $u_{i, k}=u_{i, k-1}$ for all $i \neq s, t$. For vertices $v_{s}$ and $v_{t}, \ell_{s, k}=\ell_{s, k-1}-1, u_{s, k}=u_{s, k}-1, \ell_{t, k}=\ell_{t, k-1}+1$, and $u_{t, k}=u_{i, k-1}+1$. Only the bounds of $v_{s}$ and $v_{t}$ have been changed, and their interval lengths remain unchanged because their upper and lower bounds have changed by the same amount.

Since all intervals have length $m-\sum_{i=1}^{r} \ell_{i, k-1}$ in stage $k-1$, for any hypothesis in $H\left(\mathcal{I}_{\text {bounds }}(k-1)\right)$ in which there exists a vertex $v_{i}$ that contains $u_{i, k-1}$ bodies, all other vertices must contain $\ell_{i, k-1}$ bodies. Therefore, if $\ell_{s, k-1}=0$, then any hypothesis in which $v_{s}$ contained 0 bodies in stage $k-1$ must have been false, which means that any hypothesis in which a vertex $v_{i}$ contained $u_{i, k-1}$ bodies was also false. All other hypotheses in $H\left(\mathcal{I}_{\text {bounds }}(k-1)\right)$ can be modified into hypotheses consistent with $\mathcal{I}_{\text {bounds }}(k-1)+y_{k}$ by moving one body from $v_{s}$ to $v_{t}$. For each $v_{i} \neq v_{s}$, there exists a hypothesis in $H\left(\mathcal{I}_{\text {bounds }}(k-1)\right)$ where $v_{i}$ contains $u_{i, k-1}-1$ bodies and $v_{s}$ contained one body. There also exists a hypothesis in $H\left(\mathcal{I}_{\text {bounds }}(k-1)\right)$ where $v_{i}$ contained no bodies, so when the incorrect hypotheses are discarded and a body is moved from $v_{s}$ to $v_{t}$, all intervals shorten by one body. Therefore, $\mathcal{I}_{\text {bounds }}(k)$ is constructed by setting $\ell_{t, k}=\ell_{t, k-1}+1$ and $u_{t, k}=u_{t, k-1}$. For each vertex $v_{i}$ where $v_{i} \neq v_{t}$, the bounds are constructed by setting $\ell_{i, k}=\ell_{i, k-1}$ and $u_{i, k}=u_{i, k-1}$.

Note that if $u_{t, k-1}=m$, then for all other vertices $v_{i}$, the value of $\ell_{i, k-1}$ must be 0 , as any hypothesis that places $m$ bodies into $v_{t}$ must place zero bodies in all other vertices. Therefore, the situation where a hypothesis is disqualified because a body moves into a vertex with an upper bound of $m$ bodies is a special case of the situation in the previous paragraph.

Therefore, for all $k \in \mathbb{N}$ and all $v \in V(G)$, the interval length length $(k, v)=m-$ $\sum_{i=1}^{r} \ell_{i, k}$.

Theorem 1 implies that keeping track of an upper bound for each individual vertex is redundant, as all upper bounds can be reconstructed using the lower bounds and the total number of bodies. This means that once the lower bound of a vertex $v_{i}$ and the number of bodies in $v_{i}$ are the same, we have in some sense learned all that we can from $v_{i}$. A corollary to Theorem 1 formalizes this notion. An informative observation is a sensor observation that decreases the interval length.

Corollary 2. The information state $\mathcal{I}_{\text {bounds }}(k)$ is a counting information state if and only if for each vertex $v \in V(G)$ there exists a stage $j$, where $0 \leq j \leq k$, and $v$ contains no bodies at stage $j$.

Proof. Let $d$ be some initial distribution of bodies. Suppose that there is a vertex $p$ that did not empty out during the first $k$ stages. Let $q$ be a vertex such that $q \neq p$. Now, consider an initial distribution $d^{\prime}$ that is exactly the same as $d$ except there is one more body in $q$ and one fewer body in $p$. Since, starting from initial distribution $d$, the vertex $p$ did not fully empty out in the first $k$ stages, both $d$ and $d^{\prime}$ are capable of producing $\mathcal{I}_{\text {hist }}(k)$. Note also that starting from initial distribution $d^{\prime}$, the vertex $q$ did not empty out in the first $k$ stages. Therefore, it is impossible to determine if the starting distribution was $d$ or $d^{\prime}$. Since one would get a different distribution at 
stage $k$ when starting from $d$ than one would get by starting from $d^{\prime}$, and $\mathcal{I}_{\text {hist }}(k)$ did not rule either out, that means that there is more than one hypothesis in $\mathcal{I}_{\text {bounds }}(k)$, so $\mathcal{I}_{\text {bounds }}(k)$ is not a counting information state.

In the other direction, suppose that for each vertex $v \in V(G)$, there exists a stage prior to $k$ in which $v$ was empty. If a body moves in to $v$, the lower bound on $v$ increases. If a body leaves $v$ when $v$ has a lower bound of 1 or greater, the lower bound on $v$ decreases. If a body leaves $v$ when $v$ has a lower bound of 0 , an informative observation occurs. Suppose $v$ started with $b$ bodies, emptied out at stage $j$ (where $0 \leq j \leq k$ ). Since $v$ is empty at stage $j$, if there were $a$ entries into $v$, then there were at least $a+b$ exits from $v$. Since there were at most $a$ entries into $v$ and $v$ 's lower bound was initially 0 , at most $a$ exits could have decremented the lower bound of $v$. Therefore, at least $b$ exits from $v$ were informative observations. If each vertex empties out, then each vertex produces a number of informative observations equal to the number of bodies that it initially contained. Therefore, if each vertex empties out by stage $k$, there is one informative observation for each body, which means that the interval length is 0 at stage $k$.

\section{The exponential random movement model}

In the following sections, we will determine bounds on the expected number of steps required to converge to a counting information state. In order for these bounds to be well-defined, we require a model for the movement of the bodies. We have chosen to focus on a model in which the movement of the bodies are associated with exponential random variables.

Associate each edge of the graph with a positive weight. For a body $a \in M$ contained in a vertex $v$, the amount of time $a$ spends in $v$ is described by an exponential random variable $Y(v)$ with a rate parameter proportional to the sum of the weights of the outgoing edges incident to $v$. When $a$ moves, it selects an outgoing edge incident to $v$ (the probability of an edge being selected is proportional to its weight).

One physical system that approximately produces this movement model is balls bouncing via specular bounces in polygonal regions separated by small doorways, similar to mathematical billiards. See Figure 2 for a representative environment and the distribution of the length of time a body remains in a single room between transitions.

A graph is edge weight balanced if there exists some positive constant $W$ such that for each vertex $v_{i}$, the sum of the weight of the outgoing edges of $v_{i}$ is equal to $W$. For example, an out-regular directed graph with unit edge weights is edge weight balanced. If a graph is edge weight balanced, then each body has the same probability of being the next body selected to move.

Let $C_{2}$ be a directed cycle graph on two vertices where both edges have weight 1. Given a graph $G$ and a distribution $(d, L)$ that is near-complete for a vertex $v_{i} \in$ $V(G)$, let $C_{2}(d, L)$ be a distribution and lower bound set on $C_{2}$ that is near-complete for $v_{1} \in C_{2}$ where $v_{1}$ contains the same number of bodies that $d$ places in $v_{i}$. 


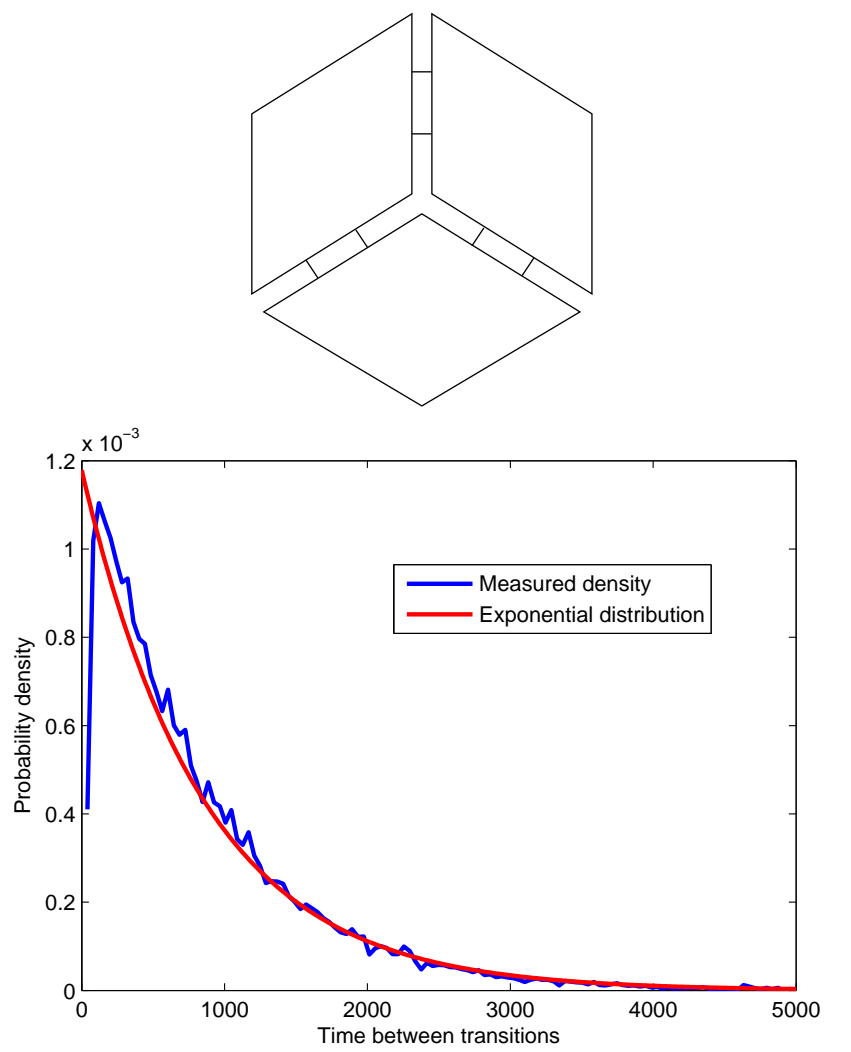

Fig. 2 [top] An environment with three symmetric regions. [bottom] The distribution of the lengths of time spent in a single region between transitions compared to an exponential random variable with the same mean. The values along the $x$-axis count simulation steps.

Let $\operatorname{Con}(G, d)$ be a random variable denoting the number of sensor observations required to converge to a counting information state in an edge weight balanced graph $G$ starting from an initial body distribution $d$ with the bodies using the exponential random movement model. We will refer to $E[\operatorname{Con}(G, d)]$ as the expected exponential convergence time, or the EE-convergence time. The word "exponential" in the term refers to the behavior of the random variables governing the motion of the bodies. The remainder of this paper will focus primarily on placing bounds on the EE-convergence time.

\section{An upper bound on the EE-convergence time}

Let graph $G$ contain $m$ bodies. For a distribution of bodies and set of lower bounds $(d, L)$, let $\operatorname{Info}(G, d, L)$ be a random variable denoting the number of stages until an informative observation occurs. Let $\beta(G)$ be the maximum of $E[\operatorname{Info}(G, d, L)]$ over all choices of $(d, L)$. 
Lemma 3. If $E[\operatorname{Info}(G, d, L)]=\beta(G)$, then $(d, L)$ is near-complete.

Proof. Suppose that the lemma is false and let $L$ be any set of lower bounds consistent with $d$ such that $\sum_{\ell_{i} \in L} \ell_{i}<m-1$ and $E[\operatorname{Info}(G, d, L)]$ is maximal. Let $L^{\prime}$ be a set of lower bounds consistent with $d$ such that $\sum_{\ell_{i}^{\prime} \in L^{\prime}} \ell_{i}^{\prime}=m-1$ and for all $1 \leq i \leq r, \ell_{i}^{\prime} \geq \ell_{i}$. Note that, because $\sum_{\ell_{i}^{\prime} \in L^{\prime}} \ell_{i}^{\prime}=m-1$, there is exactly one vertex $p$ where $p$ 's lower bound in $L^{\prime}$ is not equal to its number of bodies (the two values differ by exactly one body). Similarly, either there exists a vertex $q$ such that $q \neq p$ and $q$ 's lower bound in $L$ is also not equal to its number of bodies, or $p$ 's lower bound in $L$ differs from its number of bodies by two or more.

Let $S^{\prime}$ be the set of finite sequences of sensor observations that, starting from $L^{\prime}$ and $d$, contain exactly one informative observation, located at the end of the sequence. For $s \in S$, let $p(s)$ be the probability that sequence $s$ occurs. Note that $p(s)$ is only dependent on $s$ and $d$, not on $L^{\prime}$. Note that $E\left[\operatorname{Info}\left(G, d, L^{\prime}\right)\right]=\sum_{s^{\prime} \in S^{\prime}} p\left(s^{\prime}\right)\left|s^{\prime}\right|$. Since the interval length for $L^{\prime}$ is one, each sequence in $S^{\prime}$ must at some point move all bodies out of $p$.

Since $p$ also has a lower bound in $L$ that differs from its number of bodies, any sequence $s^{\prime} \in S^{\prime}$ contains a minimum prefix $s$ that is also an informative observation. Note that $E[\operatorname{Info}(G, d, L)]=\sum_{s^{\prime} \in S} p\left(s^{\prime}\right)|s|$. If $p$ has different lower bounds in $L$ and $L^{\prime}$, then always $s \neq s^{\prime}$, as an informative observation for $L$ will be made when $p$ empties all but one body. Otherwise, the aforementioned vertex $q$ exists, and $s \neq s^{\prime}$ if $q$ is emptied out prior to $p$ emptying out. Since $|s| \leq\left|s^{\prime}\right|$, and there exists at least one sequence where $|s|<\left|s^{\prime}\right|$, we get that $E[\operatorname{Info}(G, d, L)]=$ $\sum_{s^{\prime} \in S} p\left(s^{\prime}\right)|s|<\sum_{s^{\prime} \in S} p\left(s^{\prime}\right)\left|s^{\prime}\right|=E[\operatorname{Info}(G, d, L)]$. However, this contradicts the maximality of $E[\operatorname{Info}(G, d, L)]$.

Therefore, if $E[\operatorname{Info}(G, d, L)]=\beta(G)$, then $\sum_{\ell_{i} \in L} \ell_{i}=m-1$.

We will now obtain a slightly stronger result for $C_{2}$ that will be useful later.

Lemma 4. The expected value of $\operatorname{Info}\left(C_{2}, d, L\right)$ is maximized when $(d, L)$ is nearcomplete for some vertex $v_{1}$, and $v_{1}$ contains all $m$ bodies.

Proof. For a two vertex graph with one edge, Lemma 3 implies that $E\left[\operatorname{Info}\left(C_{2}, d, L\right)\right]$ is maximized when there exists a vertex (called $v_{2}$ ), in which the lower bound of $v_{2}$ is equal to the number of bodies in $v_{2}$, and another vertex $v_{1}$ in which the lower bound of $v_{1}$ is one less than the number of bodies in $v_{1}$. An informative observation will only occur if $v_{1}$ completely empties.

Let $d$ and $L$ be the distribution where $d$ places all $m$ bodies into $v_{1}$ and the lower bound $\ell_{1}$ is equal to $m-1$. Let $d^{*}$ be any other distribution, and let $L^{*}$ be a set of lower bounds such that $\left(d^{*}, L^{*}\right)$ is near-complete for $v_{1}$. Note that, for both $(d, L)$ and $\left(d^{*}, L^{*}\right)$, an informative observation is only possible if $v_{1}$ empties completely. However, since $d *$ places fewer bodies in $v_{1}$ than $d$ places in $v_{1}$, any sequence of sensor observations that results in an informative observation starting from $(d, L)$ must result in $\left(d^{*}, L^{*}\right)$ as an intermediate distribution and set of bounds. Therefore, $E\left[\operatorname{Info}\left(C_{2}, d, L\right)\right]=E\left[\operatorname{Info}\left(C_{2}, d^{*}, L^{*}\right)\right]+E[$ Catchup], where Catchup is a random variable denoting the number of sensor observations required to reach 
$\left(d^{*}, L^{*}\right)$ from $(d, L)$. Since Catchup is at least one (because $\left.d \neq d^{*}\right)$, we have that $E\left[\operatorname{Info}\left(C_{2}, d, L\right)\right]>E\left[\operatorname{Info}\left(C_{2}, d^{*}, L^{*}\right)\right]$.

Recall that a graph is edge weight balanced if each vertex has the same sum of weights of incident edges.

Lemma 5. If $G$ is edge weight balanced and $(d, L)$ is near-complete for $v_{i} \in V(G)$ and $\left(d_{2}, L_{2}\right)=C_{2}(d, L)$, then $E[\operatorname{Info}(G, d, L)] \leq E\left[\operatorname{Info}\left(C_{2}, d_{2}, L_{2}\right)\right]$.

Proof. An informative observation only occurs if $v_{i}$ empties completely. We will call a body movement progressive if the body moves out of $v_{i}$. We will call a body movement regressive if the body moves into $v_{i}$. We will call a body movement neutral if it is not progressive or regressive.

In $C_{2}$, neutral movements are impossible. If there are $z$ bodies in $v_{1}$, then there is a $z / m$ probability of a progressive move, and an $(m-z) / m$ probability of a regressive movement.

Since $G$ is edge weight balanced, each body has an identical probability of being selected as the next body to move. If there are $z$ bodies in $v_{i}$, then there is a $z / m$ probability of a progressive move. There is a $(m-z) / m$ probability of a regressive or neutral movement.

Therefore, for all $(d, L)$ on $G$ that are near-complete for $v_{i}$, the probability of a progressive move is the same as in $C_{2}(d, L)$, and the probability of a regressive move in $(d, L)$ is at most the probability of a regressive move in $C_{2}(d, L)$. Therefore, $E[\operatorname{Info}(G, d, L)] \leq E\left[\operatorname{Info}\left(C_{2}, d_{2}, L_{2}\right)\right]$.

Combining all of these results leads to a bound on the EE-convergence time.

Theorem 6. For any edge weight balanced graph $G$ containing $m$ bodies, and any distribution of bodies $d$, the expectation $E[\operatorname{Con}(G, d)] \leq(2 \pi)^{-1 / 2} m^{3 / 2} e^{m-\frac{1}{12 m+1}}$.

Proof. Our first task is to bound $\beta(G)$. Lemma 5 implies that $\beta(G) \leq \beta\left(C_{2}\right)$. Lemma 4 implies that $\beta\left(C_{2}\right)=E\left[\operatorname{Info}\left(C_{2}, d, L\right)\right]$ when $(d, L)$ is near-complete for a vertex $v_{1}$.

We can bound $\beta\left(C_{2}\right)$ by considering only one specific way of clearing out $v_{1}$. Suppose that, if $v_{1}$ is not cleared out in exactly $m$ turns (we will refer to these $m$ turns as a round), then the distribution is reset to a near-complete distribution for $v_{1}$ and a new round is started. Let $\gamma\left(C_{2}\right)$ be the expected number of observations required to clear $v_{1}$ under this "resetting" condition. Since the near-complete distribution has the highest expected number of observations until an informative observation, $\gamma\left(C_{2}\right) \geq \beta\left(C_{2}\right)$.

The probability of a near-complete distribution in $C_{2}$ clearing the vertex $v_{1}$ in a single round is

$$
\prod_{i=0}^{m-1} \frac{m-i}{m}=\frac{m !}{m^{m}} .
$$

Stirling's approximation yields $m ! \geq \sqrt{2 \pi m}(m / e)^{m} e^{\frac{1}{12 m+1}}$, which puts an upper bound on the expected number of rounds until $v_{1}$ empties out. This upper bound is 


$$
\frac{e^{m}}{\sqrt{2 \pi m} e^{\frac{1}{12 m+1}}} .
$$

Since each round consists of $m$ sensor observations, Equation 2 results in the following upper bound on $\gamma\left(C_{2}\right)$, which is also an upper bound on $\beta(G)$.

$$
\beta(G) \leq \beta\left(C_{2}\right) \leq \gamma\left(C_{2}\right) \leq \frac{\sqrt{m} e^{m}}{\sqrt{2 \pi} e^{\frac{1}{12 m+1}}} .
$$

Since $\beta(G)$ is the maximum expected number of stages for an informative observation, and Theorem 1 implies that there can be at most $m$ informative observations, we get that

$$
E[\operatorname{Con}(G, d)] \leq m \beta(G) \leq \frac{m^{\frac{3}{2}} e^{m}}{\sqrt{2 \pi} e^{\frac{1}{12 m+1}}}
$$

\section{Estimation of the number of moving bodies}

We have been assuming that the total number of moving bodies is known. Suppose that the number of bodies is not known in advance. By combining the results of Sections 5 with the Markov bound

$$
\operatorname{Pr}[X \geq \alpha] \leq \frac{E[X]}{\alpha}
$$

and Bayes' Theorem,

$$
\operatorname{Pr}[A \mid B]=\frac{\operatorname{Pr}[B \mid A] \operatorname{Pr}[A]}{\operatorname{Pr}[B]}
$$

we can estimate the total number of bodies in an edge weight balanced graph, assuming that we have knowledge about the prior probability distribution over the number of bodies.

Let Bdy be a random variable denoting the number of bodies in the graph $G$.

Theorem 7. Let $z$ be the number of acquired sensor observations. Let $k$ be the sum of the lower bounds after $z$ observations have been acquired. Let $j$ be an integer such that $j>k$. The following relationship holds:

$$
\operatorname{Pr}[\mathrm{Bdy}=j \mid \mathrm{Bdy}=k \cup(\mathrm{Bdy}>k \cap \operatorname{Con}(G, d) \geq z)] \leq \frac{j^{\frac{3}{2}} e^{j}}{z \sqrt{2 \pi} e^{\frac{1}{12 j+1}}} \frac{\operatorname{Pr}[\mathrm{Bdy}=j]}{\operatorname{Pr}[\mathrm{Bdy}=k]}
$$


Proof. Using Bayes' Theorem, we get that $\operatorname{Pr}[\mathrm{Bdy}=j \mid \mathrm{Bdy}=k \cup(\mathrm{Bdy}>k \cap$ $\operatorname{Con}(G, d) \geq z)]$ is equal to

$$
\frac{\operatorname{Pr}[\mathrm{Bdy}=k \cup(\mathrm{Bdy}>k \cap \operatorname{Con}(G, d) \geq z) \mid \mathrm{Bdy}=j] \operatorname{Pr}[\mathrm{Bdy}=j]}{\operatorname{Pr}[\mathrm{Bdy}=k \cup(\mathrm{Bdy}>k \cap \operatorname{Con}(G, d) \geq z)]} .
$$

Because the condition fixes the value of Bdy, the term $\operatorname{Pr}[\mathrm{Bdy}=k \cup(\mathrm{Bdy}>$ $k \cap \operatorname{Con}(G, d) \geq z) \mid \mathrm{Bdy}=j]$ can be simplified to $\operatorname{Pr}[\operatorname{Con}(G, d) \geq z \mid \mathrm{Bdy}=j]$. The Markov bound implies

$$
\operatorname{Pr}[\operatorname{Con}(G, d) \geq z \mid \operatorname{Bdy}=j] \leq \frac{E[\operatorname{Con}(G, d) \mid \operatorname{Bdy}=j]}{z} .
$$

Theorem 6 implies $E[\operatorname{Con}(G, d) \mid \mathrm{Bdy}=j] \leq \frac{j^{\frac{3}{2}} e^{j}}{\sqrt{2 \pi} e^{\frac{1}{2 j+1}}}$. Additionally, $\operatorname{Pr}[\mathrm{Bdy}=$ $k \cup(\operatorname{Bdy}>k \cap \operatorname{Con}(G, d) \geq z)] \geq \operatorname{Pr}[\operatorname{Bdy}=k]$, as the term on the right is a strictly stronger condition. Substituting these bounds into Equation 8 yields

$$
\operatorname{Pr}[\mathrm{Bdy}=j \mid \mathrm{Bdy}=k \cup(\mathrm{Bdy}>k \cap \operatorname{Con}(G, d) \geq z)] \leq \frac{j^{\frac{3}{2}} e^{j}}{z \sqrt{2 \pi} e^{\frac{1}{12 j+1}}} \frac{\operatorname{Pr}[\mathrm{Bdy}=j]}{\operatorname{Pr}[\mathrm{Bdy}=k]}
$$

Theorem 7 can also be used to give a lower bound on $\operatorname{Pr}[\mathrm{Bdy}=k \mid \mathrm{Bdy}=k \cup$ $($ Bdy $>k \cap \operatorname{Con}(G, d) \geq z)$.

Corollary 8. Let $z$ be the number of acquired sensor observations. Let $k$ be the sum of the lower bounds after $z$ observations have been acquired. Let $j$ be an integer such that $j>k$. The following relationship holds:

$$
\operatorname{Pr}[\mathrm{Bdy}=k \mid \mathrm{Bdy}=k \cup(\operatorname{Bdy}>k \cap \operatorname{Con}(G, d) \geq z)] \geq 1-\lambda,
$$

in which

$$
\lambda=\sum_{j=k+1}^{\infty} \frac{j^{\frac{3}{2}} e^{j}}{z \sqrt{2 \pi} e^{\frac{1}{12 j+1}}} \frac{\operatorname{Pr}[\mathrm{Bdy}=j]}{\operatorname{Pr}[\mathrm{Bdy}=k]} .
$$

Proof. The term

$$
\sum_{j=k+1}^{\infty} \frac{j^{\frac{3}{2}} e^{j}}{z \sqrt{2 \pi} e^{\frac{1}{12 j+1}}} \frac{\operatorname{Pr}[\mathrm{Bdy}=j]}{\operatorname{Pr}[\mathrm{Bdy}=k]}
$$

is an upper bound on the probability that $\mathrm{Bdy}>k$. Since $\mathrm{Bdy} \geq k$ is known, any remaining probability mass must belong to $\operatorname{Pr}[\mathrm{Bdy}=k \mid \mathrm{Bdy}=k \cup(\mathrm{Bdy}>k \cap$ $\operatorname{Con}(G, d) \geq z)$.

The Markov bound converges slowly. If the variance of $\operatorname{Con}(G, d)$ is relatively low, then we could obtain a faster estimation of the number of bodies by using the Chebyshev bound 


$$
\operatorname{Pr}\left[|X-E[X]| \geq \alpha \sqrt{\operatorname{Var}[X]} \leq \frac{1}{\alpha^{2}} .\right.
$$

Unfortunately, we have been unable to prove any bounds on $\operatorname{Var}[\operatorname{Con}(G, d)]$. However, simulations (in $C_{2}$ ) seem to indicate that $\operatorname{Var}[\operatorname{Con}(G, d)] \approx E[\operatorname{Con}(G, d)]^{2}$. Therefore, it is unlikely that the Chebyshev bound would produce an estimate that would converge more quickly that the Markov bound.

\section{A lower bound for the EE-convergence time}

Corollary 2 establishes that each vertex must empty out at least once in order to reduce to a counting information state. This implies that each body must move at least once. If the graph is edge weight balanced, then each body has an equal probability of being the one that moved during each sensor observation. Therefore, we can acquire a lower bound on the expected number of required sensor observations by obtaining the expected number of sensor observations required until each body has moved once. This is a restatement of the classical coupon collector's problem.

Theorem 9. For an edge weight balanced graph $G$ containing $m$ bodies, and any distribution of bodies $d$, the expectation $E[\operatorname{Con}(G, d)] \geq m H_{m}$, where $H_{m}=$ $\sum_{i=1}^{m} \frac{1}{i}=\Theta(\ln m)$.

Proof. If $j$ different bodies have moved so far, then there is a $(m-j) / m$ probability that the next body that moves is a body that moves for the first time. Therefore, if $j$ different bodies have moved so far, the expected number of stages until a new body moves is $m /(m-j)$. Summing over $0 \leq j \leq m-1$ yields $m H_{m}$.

Theorem 10. The bound in Theorem 9 is tight.

Proof. To demonstrate tightness, we must construct a graph $G$ and initial body distribution $d$ such that $E[\operatorname{Con}(G, d)]=m H_{m}$. Let $G$ be the graph consisting of $m$ disjoint, disconnected copies of $C_{2}$ with all edge weights set to 1 . Note that this graph is edge weight balanced. For the initial distribution $d$, place one body in each of the $C_{2}$ subgraphs. The information state converges to the counting information state after each vertex that initially contains a body empties out at least once. Each vertex that initially contains a body starts with only one body a piece, and each component contains only one body. Therefore, if each body moves at least once, then each vertex that contained a body in the initial distribution will have emptied out at least once. By Corollary 2, this is sufficient to demonstrate convergence to a counting information state. 


\section{Results for $C_{2}$}

Due to $C_{2}$ 's very simple structure, it is easier to analyze than general graphs. It is possible to get an exact answer for the expected number of stages until convergence for any initial distribution of $C_{2}$. Let $\operatorname{Out}(a, m)$ be a random variable denoting the number of stages it would take for vertex $v_{1}$ to empty out if $v_{1}$ started with $a$ bodies, with $m$ bodies total present in the graph (due to the symmetry in the graph, $v_{2}$ could be substituted for $v_{1}$ in the definition.

It is easy to determine the value of $\operatorname{Out}(a, m)$ with a recurrence. For base cases, we have $E[\operatorname{Out}(0, m)]=0$ and $E[\operatorname{Out}(m, m)]=1+E[\operatorname{Out}(m-1, m)]$. If there are $a$ bodies in $v_{1}$, then there is an $a / m$ probability that a body leaves $v_{1}$, and an $(m-a) / m$ probability that a body enters $v_{1}$. Therefore, when $a \neq 0$, we have

$$
E[\operatorname{Out}(a, m)]=1+\frac{a}{m} E[\operatorname{Out}(a-1, m)]+\frac{m-a}{m} E[\operatorname{Out}(a+1, m)] .
$$

To obtain a recurrence that determines $E\left[\operatorname{Con}\left(C_{2}, d\right)\right]$ for arbitrary $d$, we must add an additional term. Let $\operatorname{Out}^{\prime}(a, m)$ be a random variable denoting the number of stages required for either of the vertices to empty out, given that the vertex with fewer bodies contains $a$ bodies, and the whole graph contains $m$ bodies.

As a base case, note that when $m$ is odd,

$$
\left[\operatorname{Out}^{\prime}(\lfloor m / 2\rfloor, m)\right]=1+\lceil m / 2\rceil E\left[\operatorname{Out}^{\prime}(\lfloor m / 2\rfloor, m)\right]+\lfloor m / 2\rfloor E\left[\operatorname{Out}^{\prime}(\lfloor m / 2\rfloor-1, m)\right] \text {. }
$$

For even $m$, the equation is $E\left[\operatorname{Out}^{\prime}(m / 2, m)\right]=1+E\left[\operatorname{Out}^{\prime}(m / 2-1, m)\right]$. As in the previous function, $E\left[\operatorname{Out}^{\prime}(0, m)\right]=0$. For other $m$, the relationship is similar to Equation 15:

$$
E\left[\operatorname{Out}^{\prime}(a, m)\right]=1+\frac{a}{m} E\left[\operatorname{Out}^{\prime}(a-1, m)\right]+\frac{m-a}{m} E\left[\operatorname{Out}^{\prime}(a+1, m)\right] .
$$

Combining these two functions leads to a solution for arbitrary distributions over $\mathrm{C}_{2}$.

Theorem 11. If $d$ is a distribution of $m$ bodies over $C_{2}$, with $m>1$, that places $a$ bodies in a single vertex, and $m-a$ in the other vertex, with $a \leq m-a$, then $E\left[\operatorname{Con}\left(C_{2}, d\right)\right]=E\left[\operatorname{Out}^{\prime}(a, m)\right]+E[\operatorname{Out}(m, m)]$.

Proof. The term $E\left[\operatorname{Out}^{\prime}(a, m)\right]$ is the expected number of stages for one of the two vertices to empty out. When one vertex empties out, the other must be full, meaning that the expected number of remaining steps for the second vertex to empty out is $E[\operatorname{Out}(m, m)]$.

The authors were unable to determine a closed form solution for the expectation of either Out or Out'. However, it is not difficult to derive an exponential lower bound on $E[\operatorname{Out}(m, m)]$. Theorem 11 implies that this is also a lower bound on $E\left[\operatorname{Con}\left(C_{2}, d\right)\right]$, regardless of the initial distribution $d$. 
Theorem 12. If $d$ is a distribution of $m$ bodies over $C_{2}$, with $m>1$, then

$$
E\left[\operatorname{Con}\left(C_{2}, d\right)\right] \geq E[\operatorname{Out}(m, m)] \geq \frac{2^{m+\frac{3}{2}}}{\sqrt{\pi(m-1)}(m+1) e^{\frac{9 m-8}{(12 m-11)(3 m-3)}}}=\Omega\left(\frac{2^{m}}{m^{\frac{3}{2}}}\right) .
$$

Proof. For $0 \leq a<m$, with $m>1$, let $k_{a, m}$ be the expected number of steps required to move from a state in which $v_{1}$ contains $a+1$ bodies to a state in which $v_{1}$ contains $a$ elements. Note that $E[\operatorname{Out}(a, m)]+k_{a, m}=E[\operatorname{Out}(a+1, m)]$. Combining this identity with Equation 15 yields

$$
E[\operatorname{Out}(a, m)]=1+\frac{a}{m} E[\operatorname{Out}(a-1, m)]+\frac{m-a}{m}\left(E[\operatorname{Out}(a, m)]+k_{a, m}\right) .
$$

Combining the $E[\operatorname{Out}(a, m)]$ terms and multiplying by $m / a$ gives

$$
E[\operatorname{Out}(a, m)]=\frac{m}{a}+E[\operatorname{Out}(a-1, m)]+\frac{m-a}{a} k_{a, m} .
$$

Separating $E[\operatorname{Out}(a, m)]$ into $E[\operatorname{Out}(a-1, m)]+k_{a-1, m}$ and subtracting $E[\operatorname{Out}(a-$ $1, m)]$ from both sides yields

$$
k_{a-1, m}=\frac{m}{a}+\frac{m-a}{a} k_{a, m} .
$$

Since $(m-a) / a \geq 1$ when $a \leq m / 2$, the value of $k_{a, m}$ grows as $a$ becomes smaller when $a \leq m / 2$. Due to the left term on the right side of Equation 20, we know that $k_{\lfloor m / 2\rfloor, m} \geq 2$. Therefore,

$$
k_{0, m} \geq 2 \prod_{a=1}^{\lfloor m / 2\rfloor} \frac{m-a}{a}=2 \frac{(m-1) !}{\left\lfloor\frac{m}{2}\right\rfloor !\left\lceil\frac{m}{2}\right\rceil !} .
$$

We can use the version of Stirling's approximation found in [12] to bound the factorials. For even $m$, this becomes

$$
k_{0, m} \geq \frac{2}{m} \frac{\sqrt{2 \pi m}\left(\frac{m}{e}\right)^{m} e^{\frac{1}{12 m+1}}}{\pi m\left(\frac{m}{2 e}\right)^{m} e^{\frac{1}{3 m}}}=\frac{2^{m+\frac{3}{2}}}{\sqrt{\pi m^{3}} e^{\frac{9 m+1}{3 m(12 m+1)}}} .
$$

For odd $m$, the equation becomes

$$
k_{0, m} \geq \frac{4}{m+1} \frac{\sqrt{2 \pi(m-1)}\left(\frac{m-1}{e}\right)^{m-1} e^{\frac{1}{12 m-11}}}{\pi(m-1)\left(\frac{m-1}{2 e}\right)^{m-1} e^{\frac{1}{3 m-3}}}=\frac{2^{m+\frac{3}{2}}}{\sqrt{\pi(m-1)}(m+1) e^{\frac{9 m-8}{(12 m-11)(3 m-3)}}} .
$$

The two inequalities are almost the same, but the Equation 23 is smaller for $m>1$. 


\section{Conclusion and future work}

This paper has presented a necessary and sufficient condition for determining the distribution of a number of moving bodies in an environment made of regions separated by sensor beams. Additionally, this paper has determined bounds on the expected number of sensor observations required to determine this distribution under the exponential random movement model and shown how these bounds can be used to estimate the total number of moving bodies when this number is not known in advance.

One goal should be to expand the results of Theorems 6 and 9 to graphs that are not edge weight balanced. One possible way that this could be accomplished for Theorem 6 would be to "reset" all the moving bodies into the vertex with the smallest sum of outgoing edges, as this would most likely be the hardest vertex to empty. One could also develop specific bounds for other types of graphs and starting distributions. Theorems 10 and 12 imply that for a single graph, one starting distribution causes convergence to the counting information state in an expected polynomial number of stages, and a different starting distribution causes convergence in an expected exponential number of stages (note that the graph in Theorem 10 can be treated as $C_{2}$ if all the bodies start in one component). It would be useful to know under which conditions this polynomial-to-exponential "phase transition" occurs.

Exploiting the cover time of random walks is one technique that could be used to produce improved bounds. The cover time of a vertex $v \in V(G)$ for some graph $G$ is the expected number of steps required for a body initially placed in $v$ that is taking a random walk to reach every vertex in $G$. The cover time of the graph is the maximum cover time over all possible starting vertices. For a strongly connected graph $G$ containing $m$ bodies, one could make a secondary graph $H$ with a vertex set consisting of the length $m$ strings with characters drawn from $V(G)$. Each vertex of $H$ is an string that contains the location of each of the $m$ bodies in $G$. For $v_{1}, v_{2} \in$ $V(H)$, an edge exists between them if their corresponding strings of the vertices differ in exactly one character, and the differing character in $v_{1}$ has an edge in $G$ to the differing character from $v_{2}$. A random walk by a single body in $H$ can represent the movements of all the bodies in $G$. Since $G$ is strongly connected, so is $H$. Since for each vertex in $G$, there exists a vertex in $H$ that represents a state in which $G$ is empty. Therefore, the expected time to convergence to a counting information state in $G$ is less than the expected cover time of $H$.

For a simple example, consider the case where $G=C_{2}$. In this case, $H$ is the $m$ dimension hypercube. In order for each vertex of $G$ to empty out, two vertices of $H$ have to be reached. One is the vertex of $H$ representing the state where all bodies are in $v_{1} \in V(G)$, and the other is the vertex of $H$ representing the state where all bodies are in $v_{2} \in V(G)$. Since $H$ is an regular graph with $2^{m}$ vertices, [8] implies that the expected cover time is at most $4^{m+1 / 2}$ steps. Therefore, $E\left[\operatorname{Con}\left(C_{2}, d\right)\right] \leq 4^{m+1 / 2}$. This is not as good of a bound as the ones presented earlier in the paper, but further refinement of the technique may yield useful results. 


\section{Acknowledgements}

This work is supported in part by NSF grant 0904501 (IIS Robotics), NSF grant 1035345 (CNS Cyberphysical Systems), DARPA SToMP grant HR0011-05-1-0008, and MURI/ONR grant N0001409-1-1052. The authors thank Leonardo Bobadilla and Katrina Gossman for their assistance with this project.

\section{References}

1. J. Aslam, Z. Butler, F. Constantin, V. Crespi, G. Cybenko, and D. Rus. Tracking a moving object with a binary sensor network. In 1st international conference on Embedded networked sensor systems, pages 150-161. ACM Press, 2002.

2. Y. Baryshnikov and R. Ghrist. Target enumeration via integration over planar sensor networks. In Proceedings of Robotics: Science and Systems IV, Zurich, Switzerland, June 2008.

3. Leonardo Bobadilla, Oscar Sanchez, Justin Czarnowski, Katrina Gossman, and Steven LaValle. Controlling wild bodies using linear temporal logic. In Proceedings of Robotics: Science and Systems, June 2011.

4. M. Dehn. Papers on Group Theory and Topology. Springer-Verlag, Berlin, 1987.

5. D. B. A. Epstein, M. S. Paterson, G. W. Camon, D. F. Holt, S. V. Levy, , and W. P. Thurston. Word Processing in Groups. A. K. Peters, Natick, MA, 1992.

6. M. A. Erdmann and M. T. Mason. An exploration of sensorless manipulation. IEEE Transactions on Robotics \& Automation, 4(4):369-379, August 1988.

7. Q. Fang, F. Zhao, and L. Guibas. Counting targets: Building and managing aggregates in wireless sensor networks. Technical Report P2002-10298, Palo Alto Research Center, June 2002.

8. Uriel Feige. Collecting coupons on trees, and the cover time of random walks. Computational Complexity, 6(4):341-356, 1996.

9. B. Gfeller, M. Mihalak, S. Suri, E. Vicari, and P. Widmayer. Counting targets with mobile sensors in an unknown environment. In ALGOSENSORS, July 2007.

10. K. Y. Goldberg. Orienting polygonal parts without sensors. Algorithmica, 10:201-225, 1993.

11. W. Kim, K. Mechitov, J. Choi, and S. Ham. On target tracking with binary proximity sensors. In ACM/IEEE International Conference on Information Processing in Sensor Networks, pages 301-308. IEEE Press, 2005.

12. Herbert Robbins. A remark on stirling's formula. The American Mathematical Monthly, 62(1):26-29, 1955.

13. N. Shrivastava, R. Mudumbai, U. Madhow, and S. Suri. Target tracking with binary proximity sensors: fundamental limits, minimal descriptions, and algorithms. In Proc. 4th Internat. Conf. on Embedded Networked Sensor Systems, 2006, pages 251-264. ACM Press, 2006.

14. J. Singh, R. Kumar, U. Madhow, S. Suri, and R. Cagley. Tracking multiple targets using binary proximity sensors. In Proc. Information Processing in Sensor Networks, 2007.

15. B. Tovar, F. Cohen, and S. M. LaValle. Sensor beams, obstacles, and possible paths. In Proceedings Workshop on Algorithmic Foundations of Robotics, 2008.

16. C.-C. Wang, C. Thorpe, S. Thrun, M. Hebert, and H. Durrant-Whyte. Simultaneous localization, mapping and moving object tracking. International Journal of Robotics Research, 26(9):889-916, 2007.

17. D. B. Yang, H. H. Gonzalez-Banos, and L. J. Guibas. Counting people in crowds with a realtime network of simple image sensors. In Proc. IEEE International Conference on Computer Vision, volume 1, pages 122- 129, 2003. 